\title{
Photophysical Processes in Simple Molecules Excited by Synchrotron Radiation
}

\section{IRÈNE NENNER}

Département de Physico-Chimie, Centre d'Etudes Nucleaires de Saclay, 91191 Gif sur Yvette Cedex, France and LURE, Université de Paris-sud, Bâtiment 209c, 91405 Orsay Cedex, France

The elementary photophysical processes following the absorption of a VUV photon (above $10 \mathrm{eV}$ ) by an isolated molecule are described. Special emphasis is made on photoionization and photodissociation processes, on the basis of recent results obtained by different experimental methods associated to synchrotron radiation. Discussion is made within the frame of recent theories. Future trends are also mentioned in connection with new synchrotron sources.

\section{INTRODUCTION}

The understanding of VUV molecular absorption spectra, with synchrotron radiation, has produced important progress recently. The results from recent theoretical and experimental efforts are reported in several reviews. ${ }^{1-5}$ In this paper, the energetics and dynamics of photophysical processes occuring in simple molecules excited in the VUV range, are described and discussed. The excitation energy range is strictly confined above the first ionization potential (IP) which lies around $10 \mathrm{eV}$ photon energy for molecules which are of atmospherical interest.

It is well known ${ }^{6}$ that when the photon energy is higher than the first IP, ionization competes with other decay processes such as neutral dissociation for excitation energies up to $15 \mathrm{eV}$ above the IP (that is for photon energy of about $25 \mathrm{eV}$ ). Above this energy, ionization dominates and processes such as ionization with simultaneous 
excitation, multiple ionization, core ionization and Auger processes can occur. But these high energy processes occuring above $25 \mathrm{eV}$ will not be considered here.

The most important characteristics of an absorption or total ionization cross section spectrum (PIS) is the rich and complex structures superimposed on a continuum. These structures, known as "superexcited states," are generally assigned to Rydberg series associated with the excitation of a single inner valence shell electron (deeper than the outermost valence shell) or to high lying valence states. Their observation in PIS is associated with autoionization described as a resonant two-step ionization process. In contrast, the continuum part reflects direct ionization mechanisms which are characterized by energy thresholds, associated, to a first approximation, to the ejection of an electron from various valence shells. In addition, some broad structures called shape resonances result from the trapping of a continuum electron into a quasi-bound state supported by a potential energy barrier. They are not classified as autoionizing lines because they are described with a one-electron model just as direct ionization processes. The decay rate of all superexcited states is determined by the interaction of these quasi discrete states with the large number of continua and lies in a very wide range from $10^{11}-10^{16} \mathrm{~s}^{-1}$. This means also that line broadening varies in a wide range, i.e., from a few tenths of a meV to several $\mathrm{eV}$, as opposed to most absorption lines below IP. One of the experimental consequences is that ultrahigh resolution is not always required in VUV spectroscopy. In the $10^{-11}-10^{-13} \mathrm{~s}$ range, the lifetime is comparable with a vibrational, rotational period of the molecule (see Figure 1). Consequently, the competition between dissociation and ionization mentioned above is directly related to the existence of these superexcited states.

The decay channels of these superexcited states often leads to specific vibronic states of the products. In particular, autoionization is known to produce highly vibrationally excited states which are not accessible (Franck-Condon gaps) by direct ionization processes. These states are of particular interest because they often lead to fragmentation of the parent ion at the thermodynamic threshold. Learning about the spectroscopy and the dynamics ${ }^{7}$ of these highly excited states if of great importance for the physics of ion formation and the reactivity of ions present for example in the ionosphere and low density plasmas. 

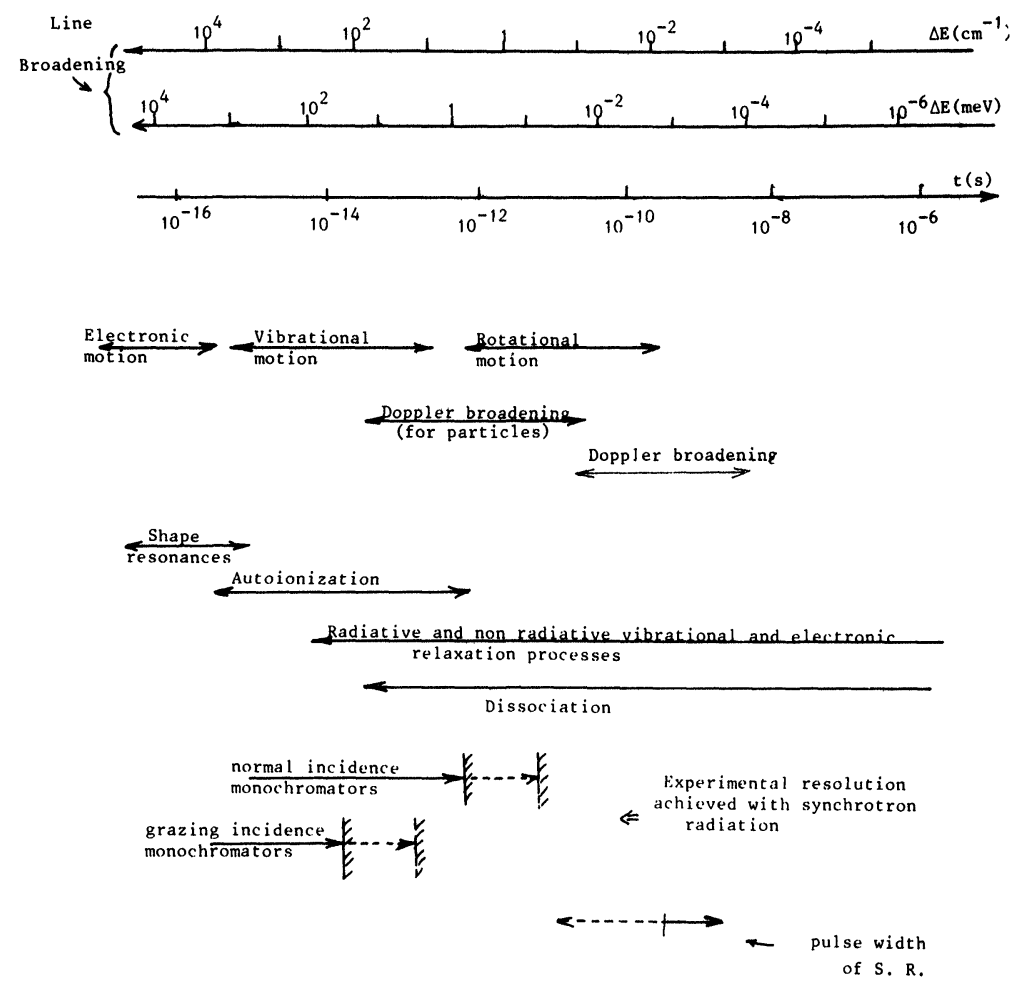

FIGURE 1 Time scale and line broadening of relevant photophysical mechanisms in the VUV excitation range. Present experimental resolutions available with normal and grazing incidence monochromators used with current S.R. sources are indicated, as well as expected time resolutions with new S.R. pulse widths (dashed lines).

Experimentally, it is very fruitful, in addition to absorption and total ionization spectra to measure partial cross sections associated to each possible decay channel, ideally for each individual rovibronic (rotational, vibrational or electronic) state of the products such as the parent and fragment ions as well as dissociation neutral fragments. Along those lines, selected experimental techniques associated to the continuous, polarized and pulsed character of synchrotron radiation have been developed. For example, partial photoionization cross sections for selected energy states of the ion were obtained either by ion fluorescence excitation spectroscopy (FES) ${ }^{8,9}$ and photoelectron 
spectroscopy (PES) at variable photon energy, ${ }^{11,12}$ called also Electron spectroscopy with Synchrotron Radiation (ESSR). Additional information on photoionization mechanisms have been obtained by Threshold photoelectron spectroscopy, ${ }^{10}$ polarization analysis of ion fluorescence ${ }^{5}$ as well as angular distributions of photoelectrons ${ }^{4,5}$ as a function of incident photon energy. For the non-ionization decay channels such as dissociation, common experiments have been limited to fragment fluorescence spectroscopy. ${ }^{13}$ In addition, many other coincidence techniques combining mass spectrometry, photoelectron spectroscopy and time resolved fluorescence spectroscopy have been also developed to study dissociative ionization or radiationless transitions in ions, but none of these aspects will be considered here.

Theoretically, one electron theories such as Multiple Scattering Model, ${ }^{4,14,15}$ Moment theory, ${ }^{16}$ Single center static-exchange theory ${ }^{17}$ have predicted a number of direct ionization partial cross sections in various diatomic and polyatomic molecules, including shape resonances. More recently, Multichannel quantum Defect theory $(\text { MODT })^{18-20}$ based on suitable ab initio calculations has been applied to selected autoionization problems in diatomic molecules.

In this paper, I will discuss successively, direct ionization with shape resonances, then autoionization and dissociation processes for diatomic and triatomic systems, based on recent results carried out by several groups using the ACO synchrotron facility at Orsay. Some future trends connected with new synchrotron sources will be also mentioned.

\section{DIRECT IONIZATION AND SHAPE RESONANCES}

The simplest direct ionization model predicts a step in the photoionization cross section occuring for each rovibronic ionic state of the ion. The amplitude of these steps is determined by the FranckCondon factors between the neutral ground state and the ionic final state. Observation of sharp steps at the first IP for most molecules is rather well established. But, the following expected steps associated to the ejection of inner valence shell electrons are rarely observed in total ionization cross sections because of the presence of autoionization. However, these steps can be observed in partial photoionization cross section measurements. 
Fluorescence excitation spectroscopy using synchrotron radiation has been shown to complement photoelectron spectroscopy to measure partial photoionization cross sections, in particular at threshold. In those experiments, one measures the variation of undispersed radiation from excited ions as a function of incident photon wavelength. Fluorescence emission spectra from different electronic states of the ion often occur in sufficiently different spectrum regions to allow direct measurements of partial photoionization cross sections, assuming an isotropic distribution of the emission. This is illustrated for the CO molecule, in Figure 2, with data taken from Ref. 21. CO has the following electronic configuration

$$
\underbrace{1 \sigma^{2} 2 \sigma^{2}}_{\begin{array}{c}
\text { core shell } \\
(1 s \text { type })
\end{array}} \quad \underbrace{3 \sigma^{2} 4 \sigma^{2}}_{\begin{array}{c}
\text { inner valence } \\
(2 s \text { type })
\end{array}} \quad \underbrace{1 \pi^{4} 5 \sigma^{2}}_{\begin{array}{c}
\text { outer valence } \\
(2 p \text { type })
\end{array}}
$$

Upon ejection of the $1 \pi$ and $4 \sigma$ electrons the $\mathrm{CO}^{+}$ion is formed in the $A^{2} \Pi$ and $B^{2} \Sigma^{+}$states respectively. Both states fluoresce to the $\mathrm{CO}^{+}, X^{2} \Sigma^{+}$ground state. $(A-X)$ and $(B-X)$ FES have been measured in a wide excitation range and compared to former FES data, ${ }^{22,23}$ PES data ${ }^{24,25}$ as well as available calculations. ${ }^{15,26,27}$ The $(A-X)$ threshold region shows a clear $v=0$ onset, but additional steps expected from higher vibrational levels of the $A$ state are not observed because of autoionizing structures. Above $20 \mathrm{eV}$, the $(A-$ $X)$ FES decreases monotonically in good agreement with theory. In contrast, the $(B-X)$ FES shows two sharp onsets at the $B, v=0$ and 1 thresholds without interference with autoionizing lines. Sharp onsets have also been observed for the $\mathrm{N}_{2} \mathrm{O}^{+} \tilde{A}^{2} \Sigma^{+}$onset, ${ }^{3,28}$ the $B$ threshold of $\mathrm{N}_{2}^{+29}$ or the $A$ and $B$ onsets of $\mathrm{CS}_{2}^{+}{ }^{30}$ In all cases, step heights vibrational distribution of a given ionic electronic state, agrees within experimental errors with Franck-Condon vibrational distributions associated with a direct ionization mechanism.

In the $\mathrm{CO}^{+}(B-X)$ FES seen in Figure 2, an intense and wide peak centered at $32 \mathrm{eV}$ has been interpreted by several theories ${ }^{15,26}$ as a shape resonance. Many molecular partial photoionization cross sections exhibit wide and intense peaks, often interpreted as shape resonances associated to the ejection of core, inner valence or outer valence electrons. ${ }^{6}$ Most of them are several $\mathrm{eV}$ wide and occur 


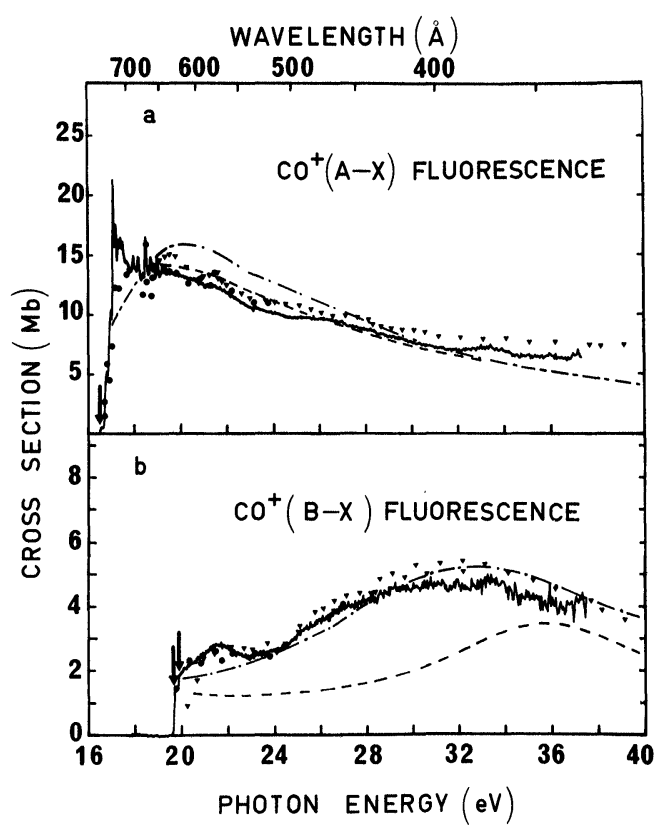

FIGURE $2 \mathrm{CO}^{+},(A-X)$ and $(B-X)$ fluorescence excitation spectra in the 16$38 \mathrm{eV}(775-325 \AA)$ excitation range. The photon bandwidth is $2 \AA$; extension of threshold regions can be found in Ref. 21; PES from Ref. 25; $\nabla$ PES results from Ref. $24 ;---x_{a}$ MSM calculations from Ref. $15 ;-.-$ Moment theory calculations from Ref. 26.

around some $10 \mathrm{eV}$ above threshold. However, definite shape resonance assignment must be accompanied by more detailed measurements. Because shape resonances originate from the existence of a potential barrier with a shape varying with internuclear distance, $, 37,33$ one expects large deviations to Franck--Condon vibrational distributions in the resonance region. This is why vibrationally resolved partial cross sections measured by ESSR have brought recently much more information on shape resonances. The clearest case of shape resonance occurs for the ejection of the outermost electron $\left(3 \sigma_{\mathrm{g}}\right)$ of nitrogen molecule. Both experimental ${ }^{31,32}$ and theoretical results ${ }^{17,33,34}$ agree on the amplitude and position. Note that vibrationally resolved measurements are not yet available for the $32 \mathrm{eV}$ peak in $\mathrm{CO}$ for a final attribution. 
However, shape resonance assignment may not be straightforward because of possible interference in the spectrum from autoionizing Rydberg states or other doubly excited states. The recent study on oxygen molecule, ${ }^{9,35,36}$ shows that the strong $3 \sigma_{g} \varepsilon \sigma_{u}$ shape resonance occurs around only $3.3 \mathrm{eV}$ above threshold (around $21.5 \mathrm{eV}$ photon energy) where intense autoionizing features also occur (see details below). Another assignment problem is the predicted strong shape resonance associated to the ejection of the outermost electron $(5 \sigma)$ of $\mathrm{CO}$. There is still some disagreement between vibrational branching ratios measured elsewhere by $\mathrm{ESSR}^{37}$ with theory, ${ }^{27}$ this may be explained by the near-by presence of an intense non-Rydberg autoionizing feature which interferes strongly with the shape resonance. Similar problems are under discussion for acetylene ${ }^{38}$ which is isoelectronic of $\mathrm{N}_{2}$ and $\mathrm{CO}$.

Several theories ${ }^{5,16,39}$ have also predicted that the anisotropy parameter $\beta$ which is measured by angular distribution of the photoelectron spectrum with respect to the polarization vector of synchrotron light may vary strongly with excitation energy along the shape resonance region. Many measurements have been reported ${ }^{40}$ even in large systems. Agreement between theory and experiment is still qualitative. Note that the energy variation of this parameter is also expected along any other autoionizing line and this additional information must be taken into account together with partial photoionization cross sections for shape resonance assignment.

\section{AUTOIONIZATION}

Generally, autoionization is considered as a two-step process. An excitation into a quasi-discrete state followed by an energy transfer from the excited ionic core to one of the photoelectrons which is then ejected. Electronic autoionization refers to an electronic relaxation of the ionic core and then two electrons are involved in the process. Vibrational or rotational autoionization refer to vibrational or rotational relaxation of the core, then a single electron is involved but these processes are associated with a departure from the BornOppenheimer approximation. Note that this very simple two-step picture is not relevant for short lived quasi-discrete state and actually, 
there is a smooth transition between real quasi-discrete states and direct ionization process.

Understanding autoionization requires both the knowledge of initial state spectroscopy (Rydberg states or others) and the dynamics of their decay. Spectroscopy of high lying Rydberg series has been done, at least partly, in many molecules by high resolution absorption, ${ }^{41}$ ionization ${ }^{42}$ spectroscopy. Unusual quantum defects, linewidth and line intensities are very common in VUV molecular spectra. However, there are very few examples where quantitative or even qualitative analysis have been made to describe these processes. The best known case fully explained by MQDT ${ }^{19}$ is the rovibrational autoionization case in hydrogen molecule just above threshold. The second example is NO just above the first IP, where MQDT ${ }^{43}$ also, has been successful to explain perturbed line intensities of Rydberg states by a coupling of these Rydberg states with dissociation continua. Besides those threshold effects concentrated only with a single electronic continuum, the structured part well above IP almost always involved electronic autoionization and only a few systems have been studied.

Figure 3 illustrates the complexity of superexcited states structures in oxygen molecule ${ }^{3}$ observed in the total photoionization cross section from IP to $26 \mathrm{eV}$ and the threshold photoelectron spectrum (TPES) in the same region of excitation. The TPES is obtained by measuring the intensity of near-threshold electrons $(\Delta E \simeq 15 \mathrm{meV})$ selected by time of flight analysis, as a function of exciting wavelength. Considering that the photon energy $E_{h \nu}$ is partitioned between the electron kinetic energy and the internal energy $E_{i}$ of the residual ion, the TPES represents the relative probability for producing ions with an internal energy $E_{i}=E_{h \nu}$. Most of the TPES features occur for $\mathrm{O}_{2}^{+}$ ion states bound in the Franck-Condon region which results from successive ejection from valence shell electrons of the $\mathrm{O}_{2}$ molecule, whose configuration is

$$
\underbrace{1 \sigma_{\mathrm{g}}^{2} 1 \sigma_{u}^{2}}_{\text {core }} \quad \underbrace{2 \sigma_{\mathrm{g}}^{2} 2 \sigma_{u}^{2}}_{\text {inner valence }} \quad \underbrace{3 \sigma_{\mathrm{g}}^{2} 1 \pi_{u}^{4} 1 \pi_{\mathrm{g}}^{2}}_{\text {outer valence }}
$$

Particular interest has been recently focused on structures occurring above $18 \mathrm{eV}$ in the PIS and the production of the $\mathrm{O}_{2}^{+}$ion states $b^{4} \Sigma_{\mathrm{g}}^{-}$ and $B{ }^{2} \Sigma_{g}^{-}$. Note that because of the open shell $1 \pi_{\mathrm{g}}$ of this molecule, 

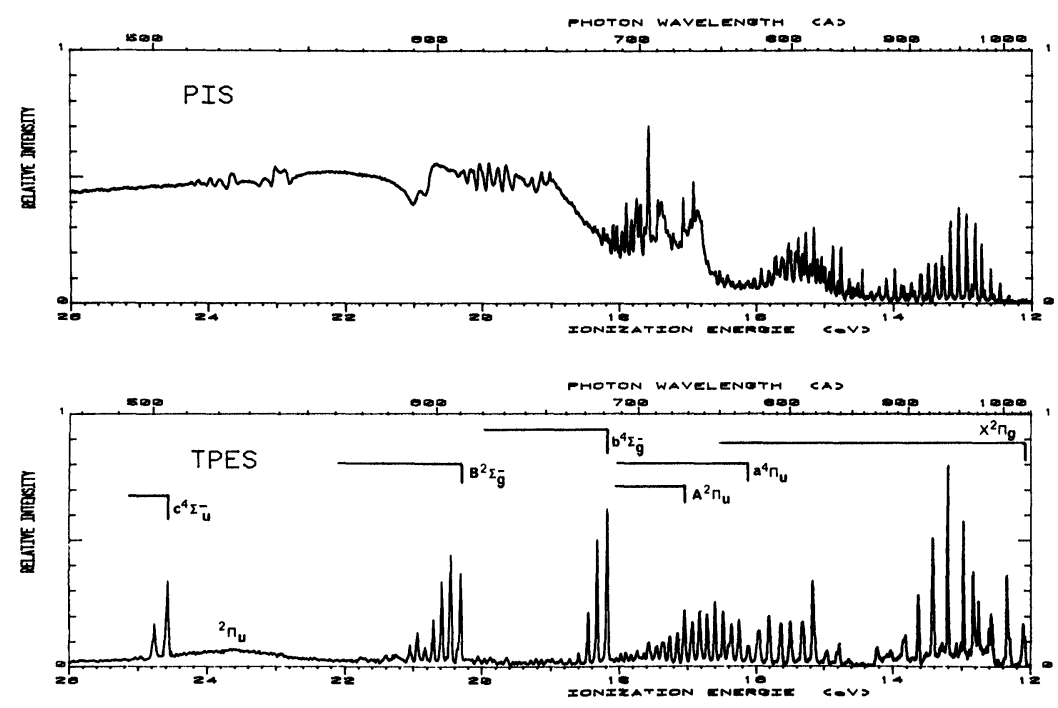

FIGURE 3 (a) $\mathrm{O}_{2}^{+}$threshold photoelectron spectrum and (b) Total ionization spectrum in the $12-26 \mathrm{eV}$ range (from Refs. 3 and 44 ).

two ionic states are produced with different binding energies, when a single inner electron is ejected. This explains why the $b$ and $B$ states originate from the ejection of a single $3 \sigma_{\mathrm{g}}$ electron. Vibronic partial cross sections for the production of the $b$ and $B$ states have been measured using the ESSR method, ${ }^{36}$ used in the constant ionic state mode, ${ }^{12}$ i.e., the electron intensity is measured as a function of photon energy, keeping the binding energy $E_{i}=E_{h \nu}-\varepsilon_{k}$ constant. A portion of this data is shown in Figure 4. One observes large structures which are vibrationally excited Rydberg series having a $B$ core but decaying primarily into the $b$ ionization continuum. Strong differences are seen considering different final vibrational ion states. Similar geometries of both autoionizing states and final ionic states, which leads to a $\Delta v=0$ propensity rule, explain the vibrational selectivity. Moreover, analysis of structures in each vibrational channel has brought satisfying explanations on these complex structures. $\mathrm{MQDT}^{36}$ based on $a b$-initio spectroscopic calculations and One Center static exchange ${ }^{17}$ calculations, reproduced in Figure 4, have shown a large coupling with the $b$ continua as well as unusually wide structures ((a), (b), (c), (d) seen 


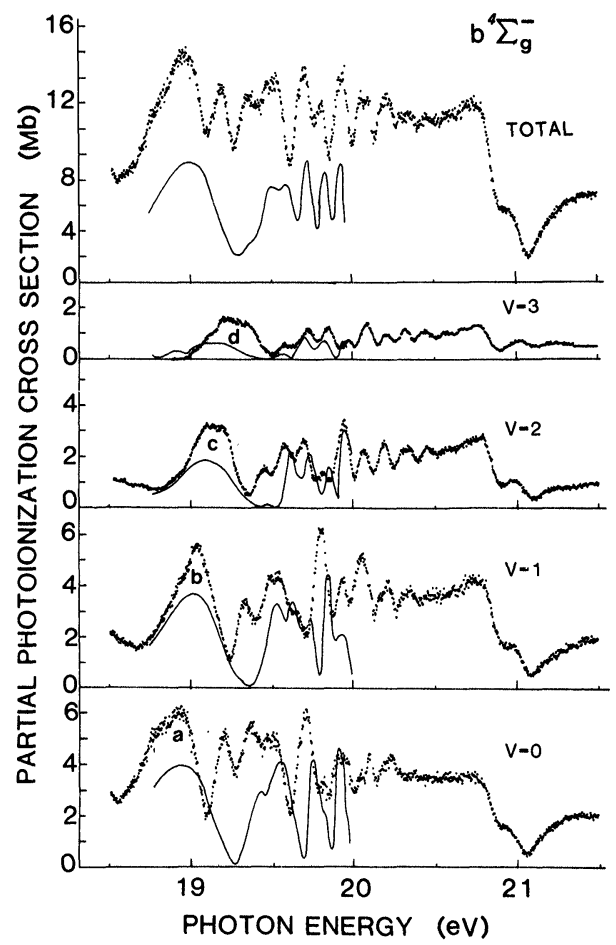

FIGURE 4 Total and vibrationally resolved partial cross sections for the $b^{4} \Sigma_{\mathrm{g}}^{-}$state of $\mathrm{O}_{2}^{+}$in the $18.17-21 \mathrm{eV}$ photon energy range, obtained by ESSR in the CIS mode. ${ }^{36}$ Dots are the experimental results. Solid lines are calculated MQDT cross sections, broadened to the experimental resolution of $0.08 \mathrm{~nm}$. (a), (b), (c) and (d) features are interpreted as due at the $4 p \sigma_{u}$ resonance states.

in Figure 4). It has been interpreted as coming from a strong valenceRydberg orbital mixing which varies with the principal quantum number. In addition the perturbing valence orbital of the $\sigma^{*}$ type, is suggested to be responsible at short internuclear distance of the shape resonance in the $B$ continuum and at large internuclear distance of the dissociative state which leads to the well known Schuman-Runge emission spectrum in neutral oxygen. This result which shows the importance of valence orbitals in the photoionization continuum, may have a general character and may help to understand strong line perturbations in the VUV range. 


\section{DISSOCIATION AND AUTOIONIZATION}

In diatomic molecules, as seen with the $\mathrm{O}_{2}$ example, dissociation induces line width and intensity perturbations, in partial cross sections. In triatomic or polyatomic molecules, additional peculiar effects have been experimentally observed. ${ }^{3,13,47}$ Namely an unexpected large number of threshold electrons have been found in Franck-Condon gaps, i.e., where direct ionization is completely negligible from the neutral ground state of the molecule. Figure 5 illustrates this situation in $\mathrm{N}_{2} \mathrm{O}$ in the excitation region between the $X^{2} \Pi$ and the $A^{2} \Sigma^{+}$ $\mathrm{N}_{2} \mathrm{O}^{+}$states. The top PIS spectrum shows numerous autoionizing structures which have been interpreted as Rydberg states converging



FIGURE 5 Total ionization and threshold electron spectra of $\mathrm{N}_{2} \mathrm{O}$ as a function of photon energy from the $X^{2} \Pi$ to $A^{2} \Sigma^{+}$states of $\mathrm{N}_{2} \mathrm{O}^{+}$(from Ref. 10); the main Rydberg series and (nd $\pi$ ) observed in the TPES are indicated. The first $\mathrm{NO}^{+}+N$ dissociation limit is indicated at the thermodynamical onset, the second one is the experimental onset of the same fragments. The $\mathrm{O}^{+}+\mathrm{N}_{2}$ experimental onset occurs at the thermodynamical limit. The dotted curve drawn on the total ion curve is an estimate of the direct ionization continuum of the $X$ state. On top are four electron time of flight spectra taken at $742,798,835$ and $893 \AA$. 
to the $A$ and $B$ states of the ion. The TPES shows in addition to the $\boldsymbol{X}$ and $\boldsymbol{A}$ vibrational bands, already observed by PES, a series of peaks in the Franck-Condon gap (region between 760 and $930 \AA$ ), arising from "resonant" (i.e., which yields near zero kinetic energy electrons) autoionization. Photoelectron spectra obtained at selected wavelengths always showed a sharp peak located at zero kinetic energy independently of photon energy. In addition, neutral dissociation compete with ionization, yielding excited and non-excited neutral fragments. Part of these processes have been measured by fragment fluorescence excitation spectrum in the same region and a summary (taken from Ref. 13) of all partial cross sections are presented in Figure 6. In this gap, all ionization processes represent $50 \%$ and dissociation $50 \%$. The first part is partitioned between $45 \%$ of direct ionization to $X$ in low vibrational levels and electronic autoionization from the Rydberg states to $X$ in low vibrational levels, and $5 \%$ of "resonant" autoionization. The second part is partitioned between dissociative states leading to fluorescing (observed) and nonfluorescing fragments. No simple diatomic-like picture could explain the large amount of threshold electrons, therefore, a model ${ }^{13}$ has been proposed based on the striking similarity between TPES and fragment FES. In the FC gap, highly vibrationally Rydberg states converging to $X$ are also excited, they autoionize into high vibrationally excited levels of $X$, with a $\Delta E=0$ propensity rule and are also mixed with dissociative states to yield fluorescing or nonfluorescing fragments.

Similar observations have been made in $\mathrm{OCS}^{44-45}$ and other systems. ${ }^{46}$ This may be viewed as an indirect observation of some sort of internal conversion between super excited states, where the vibronic density of levels is very high. However, the theory for vibrational autoionization in highly excited polyatomics has not been yet developed.

\section{FUTURE TRENDS}

The use of synchrotron radiation in photophysics will develop rapidly with the advent of new dedicated storage rings. Many possible experiments which have been largely described by Jortner and Leach, ${ }^{47}$ have not yet been done. They cover in addition to basic molecular processes medium induced molecular processes, relaxation processes 


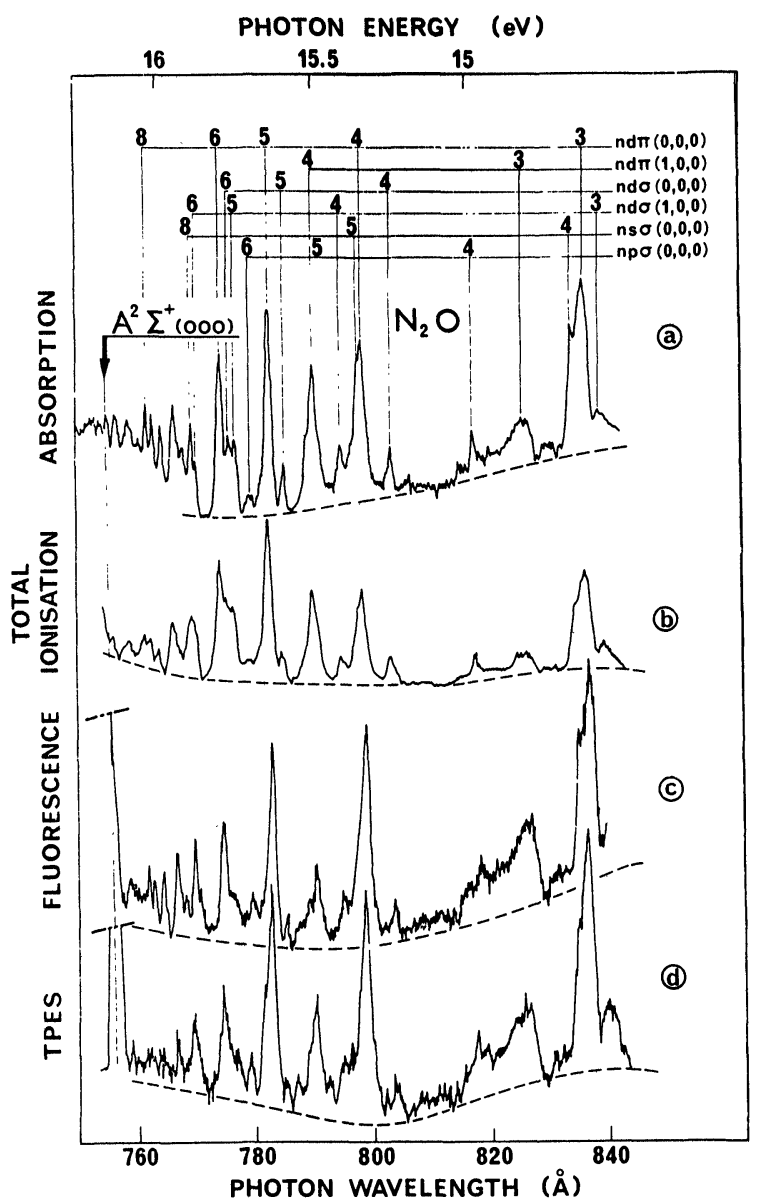

FIGURE 6 Photon excitation scans of $\mathrm{N}_{2} \mathrm{O}$ between 840 and $755 \AA$ with $0.5 \AA$ bandwidth (from Ref. 13). (a) relative variation of the absorption cross section; (b) total ion scan; (c) total fluorescence (3500-6000 ̊) excitation spectrum, coming from dissociation into excited neutral fragments; (d) threshold photoelectron spectrum.

in electric and magnetic external fields, complex molecular processes and relaxation processes in condensed phases.

Various reasons may explain this situation: lack of accessibility to storage rings, technical reasons (vacuum requirements, lack of appropriate monochromator), or psychological difficulties! Many other experiments are still not possible because present characteristics 
are largely insufficient. To overcome all the difficulties present with the electron storage rings which were designed for high energy physics, new machines have been optimized, constructed (or are under construction) and dedicated to synchrotron use. This is the case for Bessy in Berlin (Germany), Brookhaven machine and Alladin machine at Staughton (USA), SOR and Photon factory in Tsukuba (Japan), Daresbury (England) and Super-ACO in Orsay (France). Using the example of the Super-ACO project, I now summarize here the expected major improvements of importance in photophysics and photochemistry.

Firstly, the high energy of S.R. emission is chosen by an appropriate energy of circulating particles in the storage ring. More photophysicists are interested in core shell excitation in molecules, because of atomic selectivity, localization of the excitation, access to electron correlation effects etc. ... The choice of $800 \mathrm{MeV}$ will allow S.R. use up to $6 \mathrm{keV}$ photon, i.e., $K$ or $L$ excitation of atoms of the fourth row of the periodic table will be possible.

Secondly, because the electron (or positron) bunch circulating in the ring is actually the photon source, improvements can be made on its size (transverse dimensions and length) and on the density of particles. One expects with positrons an increase in brilliance of 100 over common present rings. In addition, charged particles accelerated in periodic magnetic devices (called ondulators) have been shown (depending on the number of magnetic periods) to emit light ${ }^{48,49}$ with intensities several orders of magnitude higher (up to $10^{4}$ ) than in normal dipole magnets. This is why, Super-ACO will be equipped with 6 ondulators in straight sections of the machine.

Thirdly, pulse width and repetition rate can be optimized, using an appropriate accelerating RF cavity. For a given circumference of the ring, the number of bunches $n$ gives the repetition rate. For many experiments, it must be as low as possible i.e., for $n=1$, in super ACO the maximum period will be $240 \mathrm{~ns}$. Short pulses $(\sim 0.06 \mathrm{H} \mathrm{ns})$ required for time resolved fluorescence measurements, as an example will be available. "Long" bunches ( $1 \mathrm{~ns})$ with high intensity per bunch required for electron, ion time of flight spectroscopy techniques and related coincidences measurements will also be specifically foreseen. Lastly, the additional use of lasers for their power and spectral width will also bring more refined experimental tools to this field such as two photon, two colors type of experiments. 


\section{References}

1. E. E. Koch and B. F. Sonntag, in: Synchrotron Radiation, Techniques and Applications, ed. C. Kunz (Springer-Verlag, Heidelberg, 1979) p. 269.

2. J. Berkowitz, Photoabsorption, Photoionization and Photoelectron Spectroscopy (Academic, New York, 1979).

3. P. M. Guyon and I. Nenner, Applied Optics 19, 4068 (1980).

4. T. A. Carlson, M. O. Krause, J. W. Taylor, P. R. Keller, M. N. Piancastelli, F. A. Grimm and T. A. Whitley, IEEE Transactions on Nuclear Science (1982) and references therein.

5. J. L. Dehmer, D. Dill and A. C. Parr, to appear in Photophysics and Photochemistry in the Vacuum Ultraviolet, ed. S. McGlynn, G. Findley and R. Huebner (D. Reidel Publishing Company, Dordrecht, Holland, 1983).

6. R. L. Platzman, J. Phys. Radium 21, 353 (1960).

7. T. Baer, in: Gas Phase Ion Chemistry, ed. M. T. Bowers (Academic Press, New York, 1979) Chapter 5.

8. See for example, L. C. Lee and D. L. Judge, J. Chem. Phys. 57, 4443 (1972) and references therein.

9. A. Tabché-Fouhailé, I. Nenner, P. M. Guyon and J. Delwiche, J. Chem. Phys. 75, 1129 (1981).

10. T. Baer, P. M. Guyon, I. Nenner, A. Tabché-Fouhailé, R. Botter, L. F. A. Ferreira and T. R. Govers, J. Chem. Phys. 70, 1585 (1979) and references therein.

11. P. Morin, I. Nenner, P. M. Guyon, O. Dutuit and K. Ito, J. Chim. Phys. France 77, 605 (1980).

12. P. Morin, M. Y. Adam, I. Nenner, J. Delwiche, M. J. Hubin-Franskin and P. Lablanquie, $\mathbf{N u c l}$. Inst. Meth. in press.

13. P. M. Guyon, T. Baer and I. Nenner, J. Chem. Phys. 78, 3665 (1983).

14. For example, J. L. Dehmer and D. Dill, in: Electron-Molecule and Photon-Molecule Collisions, ed. T. Rescigno, V. McKoy and B. Schneider (Plenum Press, New York, 1979) p. 183.

15. J. W. Davenport, Phys. Rev. Lett. 36, 945 (1976).

16. P. W. Langhoff, in: Electron-Molecule and Photon-Molecule Collision, ed. T. N. Rescigno, B. V. McKoy and B. Schneider (Plenum Press, New York, 1979) p. 183.

17. For example, G. Raseev, H. Le Rouzo and H. Lefebvre-Brion, J. Chem. Phys. 72, 5701 (1980); G. Raseev, H. Lefebvre-Brion, H. Le Rouzo and A. L. Roche, J. Chem. Phys. 74, 6686 (1981).

18. A. Giusti-Suzor and H. Lefebvre-Brion, Chem. Phys. Lett. 76, 132 (1980); A. Giusti-Suzor, J. Phys. B 13, 3867 (1980).

19. Ch. Jungen and D. Dill, J. Chem. Phys. 73, 3338 (1980).

20. M. Raoult, Ch. Jungen and D. Dill, J. Chim. Phys. 77, 599 (1980).

21. K. Ito, A. Tabché-Fouhailé, H. Fröhlich, P. M. Guyon and I. Nenner, J. Chem. Phys. submitted A. Tabché-Fouhailé, Doctorat d'état, Orsay (1981) unpublished.

22. G. R. Cook, P. H. Metzger and M. Ogawa, Can. J. Phys. 43, 1706 (1965).

23. L. C. Lee, R. W. Carlson and D. L. Judge, J. Phys. B 9, 855 (1976).

24. E. W. Plummer, T. Gustafsson, W. Gudat and D. E. Eastman, Phys. Rev. A 15, 2339 (1977).

25. J. A. R. Samson and J. L. Gardner, J. Electron Spectroscopy Rel. Phen. 8, 35 (1976).

26. N. Padial, G. Csanak, B. W. McKoy and P. W. Langhoff, J. Chem. Phys. 69, 2992 (1978).

27. J. A. Stephens, D. Dill and J. L. Dehmer, J. Phys. B 14, 3911 (1981).

28. A. Tabché-Fouhailé, I. Nenner, H. Fröhlich and P. M. Guyon, to be published. 
29. A. Tabché-Fouhailé, K. Ito, I. Nenner, H. Fröhlich and P. M. Guyon, J. Chem. Phys. 77, 182 (1982).

30. A. Tabché-Fouhailé, Thèse de Doctorat d'Etat, Orsay (1981), unpublished; A. Tabché-Fouhailé, M. J. Hubin-Frankskin, J. Delwiche, K. Ito, H. Fröhlich, P. M. Guyon and I. Nenner, to be published.

31. J. B. West, A. C. Parr, B. E. Cole, D. L. Ederer, R. Stockbauer and J. L. Dehmer, J. Phys. B 13, L105 (1980).

32. P. Morin, M. Y. Adam, I. Nenner, P. Lablanquie, M. J. Hubin-Franskin and J. Delwiche, to be published.

33. J. C. Dehmer, D. Dill and S. Wallace, Phys. Rev. Lett. 43, 1005 (1979).

34. R. R. Lucchese, G. Raseev and B. V. McKoy, Phys. Rev. A 25, 2572 (1982).

35. P. Morin, I. Nenner, P. M. Guyon, L. F. A. Ferreira and K. Ito, Chem. Phys. Lett. 92, 103 (1982).

36. P. Morin, I. Nenner, M. Y. Adam, M. J. Hubin-Franskin, J. Delwiche, H. LefebvreBrion and A. Giusti-Suzor, Chem. Phys. Lett. 92, 609 (1982).

37. R. Stockbauer, B. E. Cole, D. L. Ederer, J. B. West, A. C. Parr and J. L. Dehmer, Phys. Rev. Lett. 43, 757 (1979).

38. R. Unwin, I. Khan, N. V. Richardson, A. M. Bradshaw, L. S. Cederbaum and W. Domcke, Chem. Phys. Lett. 77, 242 (1981); A. C. Parr, D. L. Ederer, J. B. West, D. Holland and J. L. Dehmer, J. Chem. Phys. 76, 4349 (1982).

39. T. A. Carlson, M. O. Krause, F. A. Grimm, J. D. Allen, D. Mehaffy, P. R. Keller and J. W. Taylor, Phys. Rev. A 23, 3316 (1981).

40. T. A. Carlson, M. O. Krause, D. Mehaffy, J. W. Taylor, F. A. Grimm and J. D. Allen, J. Chem. Phys. 73, 6056 (1980).

41. For example, for $\mathrm{N}_{2}$, M. Ogawa and Y. Tanaka, Can. J. Phys. 40, 1593 (1962).

42. P. M. Dehmer and W. A. Chupka, Argonne National Laboratory, Report ANL 77-65, p. 28 (1977).

43. A. Giusti-Suzor, in: Physics of Electronic and Atomic Collisions, ed. S. Datz (North Holland, Amsterdam, 1982) p. 381.

44. J. Delwiche, M. J. Hubin-Franskin, P. M. Guyon and I. Nenner, J. Chem. Phys. 74, 4219 (1981).

45. A. Tabché-Fouhailé, M. J. Hubin-Frankskin, H. Fröhlich, K. Ito, P. M. Guyon and I. Nenner, J. Chem. Phys. submitted.

46. P. T. Murray and T. Baer, Int. J. Mass Spectrom. Ion. Phys. 30, 165 (1979).

47. J. Jortner and S. Leach, J. Chim. Phys. France 77, 1 (1980).

48. C. Bazin, M. Billardon, D. A. G. Deacon, P. Elleaume, Y. Farge, J. M. J. Madey,

49. J. M. Ortega, Y. Petroff, K. E. Robinson, M. Velghe, Physics of Quantum Electronics, Vol. 8, eds. Jacobs, Moors, Pilloff, Sargent III, Scully and Spitzer (AddisonWesley, New York, 1982) Chapter 4.

M. Billardon, D. A. G. Deacon, P. Elleaume, J. M. Ortega, K. E. Robinson, C. Bazin, M. Bergher, J. M. J. Madey, Y. Petroff, M. Velghe, Proceedings at the Bendor Free Electron Laser Conference, eds. M. Billardon and D. A. G. Deacon, Journal de Physique Colloques, to be published in 1983. 